\title{
A reply to Touring the carbon ruins: towards an ethics of speculative decarbonisation by Paul Raven and Johannes Stripple
}

The article Touring the carbon ruins: towards an ethics of speculative decarbonisation provides a thought-provoking case study on how the use of speculative design can intersect with concepts of utopianism and imaginaries. It highlights how speculative methods can be brought to bear not only on technological futures, but also speculative policymaking. The work makes a case that utopian narratives are based around assumptions that not only could there such a thing as an ideal society, but that the ideal societies portrayed or described have been achieved through 'policy interventions'. Speculative design work, and design fiction in particular, has in the past often focused heavily on technological transitions, without putting enough effort into considering the policies that must necessarily accompany them. Such work can often fall too closely into the technological utopian mindset as described in the article. This focus on the technological premise rather than the surrounding societal reactions to it can be the case even when the utopian aspects are not always to the fore. Critical and speculative design work often includes dystopian or provocative components, but again, usually at limited scale. It is vital to follow the lead of this paper and learn from utopian narrative, political speculation and imaginaries to bring not only meaning-making into our speculative work, but also the necessary collective aspect.

The case study presented is in the area of speculative decarbonisation and addresses challenges of climate change that necessitate societal action. This is a response to a problem which has been created through widespread adoption of technology (in this case the use of fossil fuels) without fully understanding or addressing the long-term consequences. It is not possible to go back in time and reinvent carbon policies with the knowledge we now have about the worldwide impacts. However, it may be possible to learn from such prior technological revolutions, and use speculative methods not only to imagine solutions to existing problems, but also as preventative methods to take technology implementations in a direction that guides away from such long-term issues. It is crucial to follow John Urry's advice (2016) and consider in terms of 'futures'; myriad possible routes that may be taken. In considering these, we should then guide ourselves towards futures with the best chance of positive societal outcomes; futures that show not just what is possible, but also what is preferable, as Voros (2003) describes. The authors highlight that such speculations may act as ethical evaluations for policy, "flagging up potential problems in implementation before they arrive, and thus allowing them to be tweaked and adjusted accordingly."

This can extend to areas beyond decarbonisation. An example of this is the work we are currently undertaking in the Ethics of $\mathrm{Al}$ in Food Data Trusts Working Group ${ }^{1}$. This interdisciplinary working group was established to consider ethical considerations relating to digital collaboration, data trusts and the use of new technologies (including Al) in the food sector (Brewer et al, 2019). Many such proposed uses raise ethical questions, which are being investigated across disciplines. For example, how might data trusts protect the rights of different organisations who might be sharing data? How do we make sure that if $\mathrm{Al}$ algorithms are used to process the data and make recommendations and predictions, that they are not biased against certain organisations or individuals? How do we ensure that these systems are transparent and have accountability built into them? What does it mean to have Al that is 'responsible'? Such questions are partly related to the technology, but are more fundamentally considerations of policy and governance. The introduction of Al technology into complex systems with high criticality, such as food supply chains, must be considered extremely carefully. Using tools such as speculation and design fiction can help concretise what may otherwise

${ }^{1}$ http://imagination.lancaster.ac.uk/update/using-design-fiction-to-think-about-food-data-ai-and-ethics/ 
be abstract concepts. For this reason, as part of the project we have developed a series of design fiction objects to represent a plausible future implementation of a food data trust using $\mathrm{Al}$, to examine ethical implications from multiple angles. This is an example of the type of speculative work Raven and Stripple highlight, which proposes to ask, "assuming a certain set of policies and technological transitions are successfully enacted in a certain location by a certain date in the future, what are the consequences, and how might life look and feel and mean to ordinary people living there?"

The next step is to consider how such tools and methods can be utilised beyond the research sphere and more directly implemented in terms of policymaking. In recent years, both design researchers and policy practitioners have shown growing interest in the role that design and design research may play in policy development. There is an increasing use of design methods by governments in the policymaking process (Whicher, 2020). Design has a particular suitability for addressing hard to define, 'wicked' problems and in visualising complexities, which characterise many policy challenges. Imagination Lancaster, the design-led research group at Lancaster University, are at the forefront of this work with a dedicated design for policy research group, which is undertaking a range of work, for example looking at how design methods are currently used, and might be further incorporated, into the work of local government.

A further strength of design for policy is its capacity to be participatory and inclusive. In considering speculative design work alongside science fictional utopian exploration, it is worth focusing a little more closely who in particular initiated the critical utopian surge in science fiction. It is notable that it is Le Guin and Delaney who are specifically mentioned as playing a key role in the 'New Wave' of science fiction, as they both belong to marginalised groups and thus represent a point of difference from the straight, white men who dominated the previous wave. The history of science fiction is intertwined with who gets the opportunity to imagine these futures, and who gets to be involved in their construction. It is interesting to consider whether the presumed 'inevitable failure' of a utopian ideal or perfected society by these authors may be influenced by their acknowledgement of the necessary heterogeneity of human culture and society.

It may be beyond the scope of this work to explore the resurgence of critical utopian science fiction in the 2010s, but it is worth considering in the light of the above that that this resurgence has been accompanied by a move towards greater diversity in terms of authors, perspectives and representation within texts. New sub-genres developed and emerged in this decade, such a growing interest in Africanfuturism, and the rise of climate-fiction (cli-fi) which Christina Orlando of publisher Tor.com noted "does seem mostly written by marginalized voices, by women, and by people of color who are starting to already see the effects of climate change in their communities" (Schnelbach et al, 2019). These trends spotlight speculations that have greater inclusivity, and input from individuals representing marginalised communities, thus a new way of questioning whose future is being represented. The authors note the limited diversity accessible to [project] due to its specific geographic situatedness. This is a limiting factor but not one that reduces the importance or significance of this work. However, it must be paired with similar endeavours that do look more broadly at broader perspectives and global framings that give alternate views on the nature of such processes and policies.

In undertaking such work, it is crucial to consider 'how the future could be' as much as 'how the future will be'. And that 'could', must include both positive and negative tellings, utopia and dystopia, in order to chart a course to a more preferable future; likely to be one that cannot be predicted. Ethical questions are not simply attendant on speculative projects, but must be a 
fundamental aspect at their core, as we use speculation to support policymakers in interrogating the ethical consequences of their policy development and implementation.

\section{References}

Brewer, Stephen., Pearson, Simon., Pauli, Michelle., Leveson-Gower, Henry., Frey, Jeremy., Maull, Roger., ... Bidaut, Luc. (2019). Digital collaboration in the food and drink production supply chain. Zenodo. http://doi.org/10.5281/zenodo.3368237

Raven, P., and Stripple, J. (2021) Touring the carbon ruins: towards an ethics of speculative decarbonisation. Global Discourse

Schnelbach, L. Orlando, C., Zutter, N., and Sweeney, R. (2019) 2010-2019: A Decade of Change in Science Fiction \& Fantasy. Tor.com https://www.tor.com/2019/12/19/2010-2019-a-decadeof-change-in-science-fiction-fantasy/ Last accessed 2 Dec 2020

Urry, J. (2016) What is the Future?, John Wiley \& Sons.

Voros, J. (2003). A generic foresight process framework. foresight.

Whicher, A. (2020) AHRC Design Fellows Challenges of the Future: Public Policy.

https://www.designresearchforchange.co.uk/wp-content/uploads/2020/08/PUBLICPOLICY2.pdf Last accessed 3 Dec 2020 